\title{
A SILICON, CARBON AND DEUTERIUM NMR INVESTIGATION OF MOLECULAR TEMPLATING IN AMORPHOUS SILICAS
}

\author{
(1) Sandia National Laboratories, Albuquerque, NM 87185-1407 \\ (2) University of Missouri, Rolla, MO 65401 \\ (3) University of New Mexico, Albuquerque, NM 87131.
}

R. A. Assink (1); C. A. Click (2), T. M. Alam (1), C. J. Brinker (1,3) and S. J. Naik (3)

\begin{abstract}
The precise pore sizes defined by crystalline zeolite lattices have led to intensive research on zeolite membranes. Unfortunately zeolites have proven to be extremely difficult to prepare in a defect-free thin film form needed for membrane flux and selectivity. We introduce tetrapropylammonium TPA (a structure directing agent for zeolite ZSM-5) into a silica sol and exploit the development of high solvation stresses to create templated amorphous silicas with pore apertures comparable in size to those of ZSM-5. ${ }^{29} \mathrm{Si}$ and ${ }^{2} \mathrm{H}$ NMR experiments were performed to evaluate the efficacy of our templating approach. The ${ }^{29} \mathrm{Si}$ NMR spectrum of the silica matrix was observed by an intermolecular cross-polarization experiment between the ${ }^{1} \mathrm{H}$ nuclei of TPA and the ${ }^{29} \mathrm{Si}$ nuclei in the silica matrix. The efficiency of the cross-polarization interaction was used to investigate the degree to which the matrix formed a tight cage surrounding the template molecule. Normally prepared xerogel materials exhibited only weak interactions between the two sets of nuclei. Drying under reduced pressure, where solvation stresses are maximized, resulted in significantly increased interactions. Analogous materials were prepared using fully deuterated TPA. The ${ }^{2} \mathrm{H}$ NMR wideline spectra consisted of a partially narrowed resonance, corresponding to template molecules which were undergoing restricted rotational motion, and an isotropically narrowed resonance, corresponding to molecules which were undergoing rapid rotational motion. The number of isotropically rotating template molecules decreased for specimens dried under reduced pressure, consistent with improved templating of amorphous silica by TPA.
\end{abstract}

\section{INTRODUCTION}

Zeolite membranes have received considerable attention for gas separation and pervaporation applications [1-3] because of their ordered and uniform pore structure. Zeolites are crystalline materials, however, and difficult to prepare as defect-free thin films needed for separation applications. A molecular templating approach is used to form well-defined porosity in amorphous silica matrices prepared by the sol-gel method. The tetrapropylammonium (TPA) cation, the structure-directing agent in ZSM-5 synthesis, was incorporated as a template within a sol-gel silica matrix. We exploit the development of high solvation stresses to create templated amorphous silicas with pore apertures comparable in size to those of ZSM-5. Organic-inorganic hybrid materials are formed, which on calcination result in TPA-templated microporous silica (Figure 1). The corresponding membranes are ultrathin, have low defect density and show molecular sieving behavior. This paper discusses several approaches based on NMR 


\section{DISCLAIMER}

Portions of this document may be illegible in electronic image products. Images are produced from the best available original document. 
spectroscopy to characterize the effectiveness of the templating process. Development of a convenient method to measure templating effectiveness will aid in optimizing the synthetic process.
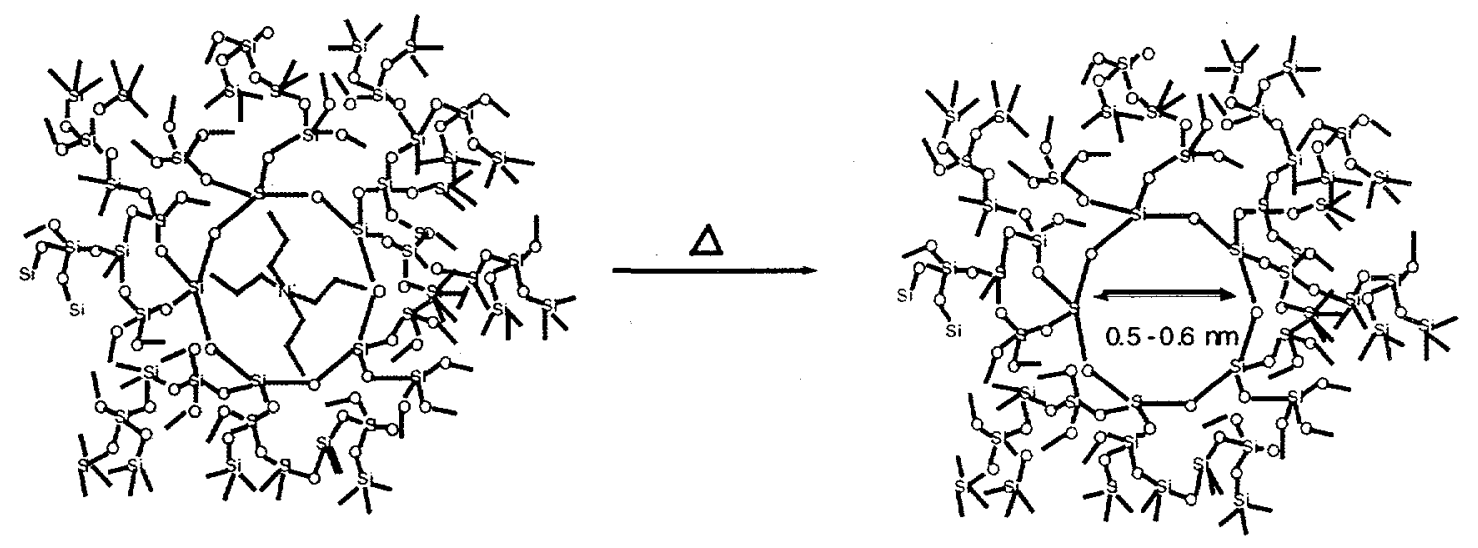

Figure 1. Schematic of TPA templating in sol-gel silica. Organic-inorganic hybrid silica formed by incorporation of TPA within a sol-gel silica matrix (left), and TPAtemplated microporous silica formed after removal of TPA by calcination (right).

The cross-polarization efficiency between two nuclei, ${ }^{\prime} \mathrm{H}$ and $\mathrm{X}$, where in this study $\mathrm{X}$ is either ${ }^{29} \mathrm{Si}$ or ${ }^{13} \mathrm{C}$, depends on the strength of the internuclear interactions and the time dependence of the fluctuations in the internuclear interactions [4]. The buildup in polarization of the $\mathrm{X}$ nuclei is most efficient when the internuclear distances are short, with the crosspolarization rate proportional to the inverse of the ${ }^{1} \mathrm{H}-\mathrm{X}$ distance raised to the sixth power. Simplified expressions for the time dependence of the fluctuations show that the crosspolarization rate is also proportional to the correlation time constant, $\tau_{c}$, describing the interaction fluctuations. Thus relatively rigid, slow moving molecules with long correlation time constants cross-polarize most efficiently. Templating effectiveness, the degree to which the matrix forms a tight cage surrounding the template molecule, is expected to affect both the internuclear distances and the correlation time constant used to describe the intermolecular crosspolarization experiment. As the templating effectiveness is increased, both factors will change so as to increase the cross-polarization efficiency. Thus overall cross-polarization efficiency can be used to measure the relative templating effectiveness.

In this study we have prepared two silica matrices in which TPA was used as a templating molecule. For bulk xerogels, slow gelation and drying allow significant time for condensation reactions to occur. This limits the extent of collapse of the gel network in response to the capillary pressure during drying. For thin film xerogels, solvent evaporation and drying occur simultaneously on a short time scale. These materials are therefore expected to show a greater extent of shrinkage of the network around the organic template molecules. We find that the thin film sample exhibits increased ${ }^{1} \mathrm{H}^{29} \mathrm{Si}$ intermolecular cross-polarization efficiency corresponding to improved templating effectiveness. Additional ${ }^{1} \mathrm{H}^{13} \mathrm{C}$ intramolecular cross-polarization experiments show that the changes in efficiency are primarily related to changes in the correlation time describing the rotational motion of the TPA molecule. The relationship between templating efficiency and the rotational motion of the TPA molecule was confirmed by ${ }^{2} \mathrm{H}$ wideline NMR spectroscopy. 


\section{EXPERIMENTAL SECTION}

Synthesis. Precursor silica sols were prepared by a two-step acid catalyzed hydrolysis and condensation process. Tetraethylorthosilicate (TEOS, 98\%, Aldrich), ethanol, deuterated water $\left({ }^{2} \mathrm{H}_{2} \mathrm{O}, 99.9\right.$ atom\% ${ }^{2} \mathrm{H}$, Cambridge Isotope Laboratories) and $\mathrm{HCl}$ were initially refluxed at $60^{\circ} \mathrm{C}$ for $90 \mathrm{~min}$. In the second step, additional ${ }^{2} \mathrm{H}_{2} \mathrm{O}$ and $\mathrm{HCl}$ were added to make up the final mole ratio of $1.0: 3.8: 5.0: 0.004$ (TEOS:EtOH: ${ }^{2} \mathrm{H}_{2} \mathrm{O}: \mathrm{HCl}$ ) and the solution was shaken for 15 minutes at room temperature. Tetrapropylammonium bromide (TPABr, 98\%, Aldrich or perdeuterated $\mathrm{TPABr}$, Isotec) was added as $4 \%$ by weight of the silica sol.

Bulk xerogel samples were prepared by gelation and slow drying of these composite sols at $50^{\circ} \mathrm{C}$. These were then ground to a fine powder. For preparing xerogels in thin film form, the sols were diluted with ethanol in the ratio 2:1 (vol. EtOH : vol. sol). These diluted sols were then cast as thin films in glass petridishes and dried at room temperature. Both the bulk and thin film xerogels were finally dried at $150^{\circ} \mathrm{C}$ for 3 hours.

Spectroscopy. The ${ }^{29} \mathrm{Si}$ and ${ }^{13} \mathrm{C}$ spectra were recorded on a Bruker AMX-400 spectrometer at 79.5 and $100.6 \mathrm{MHz}$, respectively, using $7 \mathrm{~mm}$ zirconate rotors spinning at $4 \mathrm{kHz}$. The ${ }^{2} \mathrm{H}$ spectra were recorded at $61.4 \mathrm{MHz}$ on the same spectrometer using a nonspinning $4 \mathrm{~mm} \mathrm{CP}$ MAS probe. A quadupole-echo pulse sequence with a $2 \mu$ s pulse $\left(90^{\circ}=4 \mu s\right)$ and a $60 \mu$ s delay between pulses was used.

\section{RESULTS AND DISCUSSION}

The ${ }^{1} \mathrm{H}^{29} \mathrm{Si}$ NMR intermolecular cross-polarization spectra of the bulk and thin film xerogels for a cross-polarization contact time of $3 \mathrm{~ms}$ are shown in Figure 2. This contact time is near the range of maximum intensity for each of the samples. The signal intensity of the bulk samples is less than that of the thin film sample. Since this difference is critical for our analysis, the experiments were repeated with repacked sample rotors and with careful tuning of the probe before each experiment. The relative areas of the resonances for each sample, normalized by their respective weights, are shown in the Table I. We were concerned that the two methods of preparation may result in differing concentrations of TPA molecules. The apparent difference in cross-polarization efficiencies could then be attributed to a difference in the number of TPA hydrogens that are available to cross-polarize the silicon nuclei. The relative TPA concentration in each of the samples was determined from direct, single pulse ${ }^{13} \mathrm{C}$ experiments. Delay times of $5 \mathrm{~s}$ and longer were found to provide spectra of constant intensity. The total intensities of spectra recorded at delay times of 10 and $20 \mathrm{~s}$ were combined and again normalized by the appropriate sample weights. The concentrations of TPA in the two samples provide a small correction factor shown in Table $\mathrm{I}$.
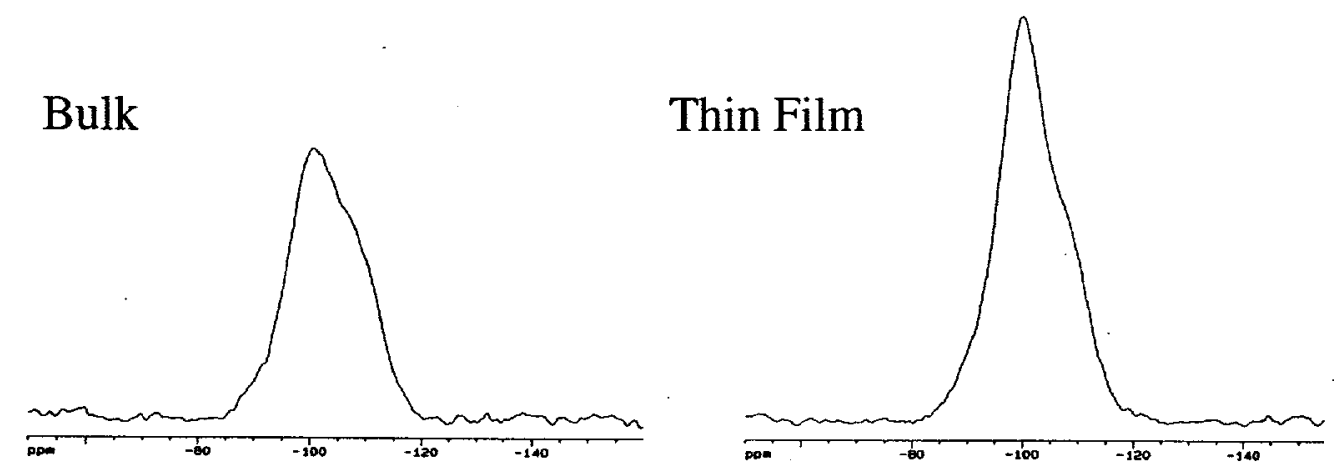

Figure 2. The intermolecular $1 \mathrm{H}-29 \mathrm{Si}$ cross-polarization spectra of the bulk and thin film xerogels recorded with a 3 ms contact time. 
Table I. Spectral Areas for ${ }^{1} \mathrm{H}_{-}{ }^{29} \mathrm{Si}$ intramolecular and ${ }^{1} \mathrm{H}^{-}{ }^{13} \mathrm{C}$ intermolecular crosspolarization experiments. All entries have been normalized by sample weights.

\begin{tabular}{|c|c|c|c|}
\hline \multicolumn{4}{|c|}{$\begin{array}{l}{ }^{1} \mathrm{H}-{ }^{29} \mathrm{Si} \text { Intramolecular Cross-Polarization Spectral Areas } \\
\text { 3ms Contact Time }\end{array}$} \\
\hline & Bulk & Thin Film & $\begin{array}{l}\text { Normalized } \\
\text { TPA conc. }\end{array}$ \\
\hline Trial \#1 & 1.00 & 1.35 & 1.36 \\
\hline Trial \#2 & 1.00 & 1.31 & 1.32 \\
\hline \multicolumn{3}{|c|}{ Average } & 1.34 \\
\hline \multicolumn{4}{|c|}{$\begin{array}{l}{ }^{1} \mathrm{H}-{ }^{13} \mathrm{C} \text { Intermolecular Cross-Polarization Spectral Areas } \\
\text { 1ms Contact Time }\end{array}$} \\
\hline Trial \#1 & 1.00 & 1.30 & \\
\hline
\end{tabular}

More effective templating leads to greater intermolecular cross-polarization efficiency. The relationship between templating and intermolecular cross-polarization depends on two factors: (1) better templating reduces the distance between the hydrogen nuclei on the TPA molecule and the silicon nuclei in the silica matrix and (2) better templating restricts the overall rotational motion of the TPA molecule and thus increases the correlation time constant describing the internuclei fluctuations. Each of these effects predicts an increase in ${ }^{1} \mathrm{H}-{ }^{29} \mathrm{Si}$ cross-polarization efficiency with increasing templating effectiveness. The ${ }^{1} \mathrm{H}^{2}{ }^{29} \mathrm{Si}$ crosspolarization experiment by itself, however, cannot determine the relative contributions of these two factors.

The ${ }^{1} \mathrm{H}_{-}{ }^{13} \mathrm{C}$ cross-polarization spectra of TPA were recorded in order to examine the intramolecular cross-polarization efficiency of the TPA molecule. Spectra with a contact time of $1 \mathrm{~ms}$, near the maximum cross-polarization efficiency, were recorded. Spectral intensities showed that the intramolecular cross-polarization efficiency of the thin film sample is greater than that of the bulk xerogel. The results, normalized for slightly differing sample weights, are shown in Table I. Under identical spectral conditions, the thin film sample has $30 \%$ greater polarization compared to the bulk xerogel. The increase in polarization must be attributed entirely to an increase in the correlation time constant describing the change in orientation of the $\mathrm{C}-\mathrm{H}$ vector with respect to the external magnetic field. Changes in internuclear distances could not contribute to this increase since the distances for intramolecular cross-polarization processes are identical for the two samples. The difference in effective correlation times depends on the difference in the overall tumbling of the TPA molecule. The ${ }^{1} \mathrm{H}-{ }^{13} \mathrm{C}$ cross-polarization experiment demonstrates that these motions are more restricted in the thin film sample.

The rotational correlation times describing the fluctuations of ${ }^{1} \mathrm{H}^{13} \mathrm{C}$ interactions in the intramolecular experiments should also modulate the ${ }^{1} \mathrm{H}^{29} \mathrm{Si}$ interactions in the intermolecular experiments. The rotational motion of the TPA molecule would move a hydrogen nucleus completely in or out of the sphere of influence of a silicon nucleus fixed in the silica matrix. Thus, we expect that the decrease in rotational mobility of the TPA molecule in the thin film sample accounts not only for the increased efficiency of the ${ }^{1} \mathrm{H}^{-13} \mathrm{C}$ cross-polarization 
experiment, but also for much of the increased efficiency of the ${ }^{1} \mathrm{H}-{ }^{29} \mathrm{Si}$ cross-polarization experiment.

In order to confirm the relationship between templating efficiency and rotational motion of the TPA molecule, we recorded the ${ }^{2} \mathrm{H}$ wideline spectra of samples which had been prepared with perdeuterated TPA. The spectra of the bulk and thin film samples are shown in Figure 3. The thin film sample consists of two sets of partially narrowed resonances. The resonance with a splitting of approximately $112 \mathrm{kHz}$ and an asymmetry parameter of 1.0 corresponds to deuterium nuclei attached to methylene carbons. The types of restricted motions in a methylene chain that can give rise to this lineshape have been discussed by Huang [5] and Hirschinger [6]. The resonance with a splitting of approximately $56 \mathrm{kHz}$ corresponds to the rapidly rotating methyl groups [7]. The spectrum of the bulk sample consists of these same two resonances and an additional narrow resonance corresponding to isotopically averaged interactions. The narrow component, corresponding to $10 \%$ of the TPA molecules in the bulk sample, represents templating molecules that are undergoing rapid overall tumbling. The presence of freely rotating TPA molecules in the bulk sample implies that the templating process is not as effective for this material.

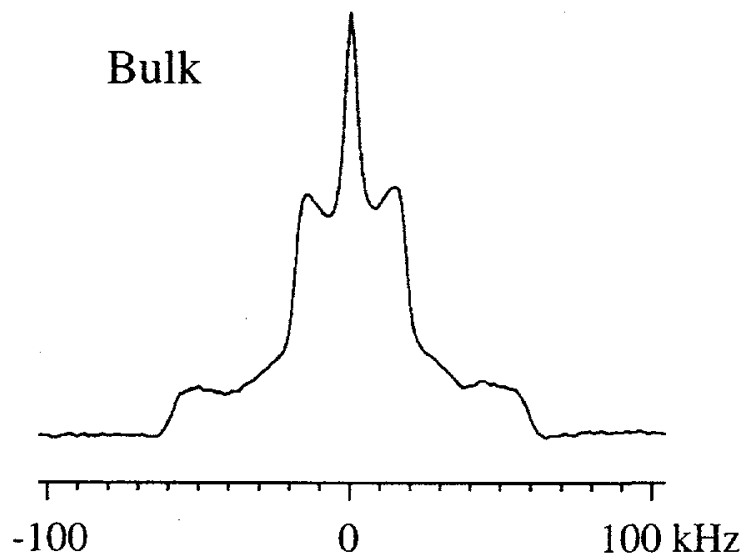

\section{Thin Film}

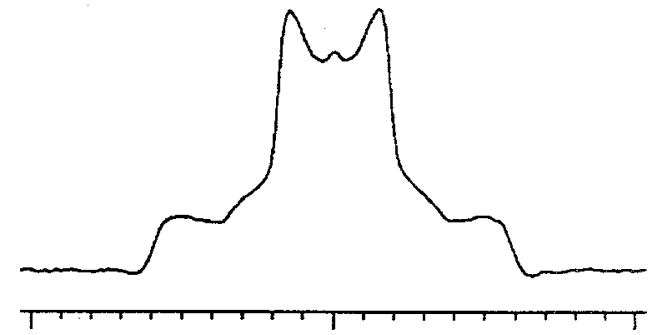

$-100$

0

$100 \mathrm{kHz}$

Figure 3. The ${ }^{2} \mathrm{H}$ wideline spectra of the bulk and thin film xerogels.

\section{SUMMARY}

We have found the intermolecular ${ }^{1} \mathrm{H}^{29} \mathrm{Si}$ cross-polarization efficiency increases during sol-gel thin film formation indicating an improved effectiveness of molecular templating. Much of the increased cross-polarization efficiency can be attributed to a reduction in the rotational mobility of the TPA templating molecule. Since the rotational motion and templating efficiency are so closely coupled, these results suggest the ${ }^{1} \mathrm{H}-{ }^{13} \mathrm{C}$ cross-polarization efficiency can be used to measure templating efficiency. Alternatively, if deuterated TPA is used in the synthesis, the deuterium lineshape provides a convenient measure of the TPA rotational motion. These probes can be used to guide and optimize the preparation of membranes by the templating process.

Sandia is a multiprogram laboratory operated by Sandia Corporation, a Lockheed Martin Company, for the United States Department of Energy under Contract DE-AC04-94AL85000. 


\section{REFERENCES}

1. T. Bein, Chem. Mater. 8, p.1636 (1996).

2. M. J. Den Exter, J. C. Jansen, J. M. Van De Graff, F. Kapteijn, J. A. Moulijn, and H. Van Bekkum, Stud. Surf. Sci. Catal. 102, p.413 (1996).

3. M. Matsukata, E. Kikuchi, Bull. Chem. Soc. Jpn. 70, p. 2341 (1997).

4. M. Mehring in NMR- Basic Principles and Progress, Vol. 11, edited by P. Diehl, E. Fluck, and R. Kosfeld (Springer-Verlag, Berlin 1976), p.112-153.

5. T. H. Huang, R. P. Skarjune, R. J. Wittebort, R. G. Griffin, and E. Oldfield, J. Am. Chem. Soc. 102, p.7379 (1980).

6. J. Hirschinger and A. D. English, J. Magn. Reson. 85, p.542 (1989).

7. G. L. Hoatson and R. L. Vold in NMR-Basic Principles and Progress, Vol. 32, edited by P. Diehl, E. Fluck, H. Gunther, R. Kosfeld, J. Seelig, and B. Blumich (SpringerVerlag, Berlin (1994), p.42-44. 